\title{
Clinical Analysis of Community-Acquired Pneumonia in the Elderly
}

\author{
Yoshihiro Kobashi, Niro Oкimoto, Toshiharu Matsushima* and Rinzo Soejima**
}

\begin{abstract}
Objective To evaluate the clinical features, etiology, and outcome of patients over 65 years old hospitalized for community-acquired pneumonia.

Patients Eighty-four patients (50 males, 34 females) hospitalized for community-acquired pneumonia in Kawasaki Medical School Kawasaki Hospital between April 1998 and March 2000.

Results Most of the patients had respiratory symptoms or signs, but over one-third also had atypical symptoms of pneumonia such as dyspnea, consciousness disturbance, and gastrointestinal symptoms. The causative microorganisms were identified in $48 \%$ of these patients. Streptococcus pneumoniae $(13 \%)$, respiratory viruses $(13 \%)$, Haemophilus influenzae (8\%) and Mycobacterium tuberculosis (8\%) were frequently identified, but Mycoplasma pneumoniae was less frequently noted in the elderly. Double infection was recognized in $19 \%$ and a combination of some virus and bacteria in $13 \%$. Treatment consisted of the administration of second or third generation cephalosporin antibiotics intravenously, because antibiotics had already been preadministered in $39 \%$. The prognosis was poor (mortality rate $9 \%$ ) for the elderly with community-acquired pneumonia despite mechanical ventilation in $8 \%$.

Conclusions Although the range of microorganisms causing community-acquired pneumonia differed slightly from that in previous reports; namely, lower frequency of Chlamydia pneumoniae and Legionella pneumophila, it is suggested that the initial antibiotic treatment should always cover $S$. pneumoniae and $\boldsymbol{H}$. influenzae. In addition, since a prevalence of virus infections related to the increase in community-acquired pneumonia in the elderly was found in this study, the routine use of influenza vaccine and pneumococcal vaccines in the elderly is recommended to reduce the high mortality rate.
\end{abstract}

(Internal Medicine 40: 703-707, 2001)
Key words: clinical features, elderly patients, causative microorganisms, treatment

\section{Introduction}

Regarding community-acquired pneumonia, guidelines were proposed by the American Thoracic Society (ATS) in 1993, the British Thoracic Society (BTS) in 1993, the Infectious Diseases Society of America (IDSA) in 1998, and the Japanese Respiratory Society in 2000 (1-4). However, only in the guidelines of the ATS has there been mention of etiological microorganisms and treatment in the elderly with community-acquired pneumonia. In those guidelines, it was noted that Streptococcus pneumoniae, respiratory viruses, aerobic gram-negative bacilli and Staphylococcus aureus appeared to be the most common pathogens in community-acquired pneumonia in outpatients without comorbidity and/or who were 60 years of age or older. The therapies recommended were second generation cephalosporin or TMX/SMX or $\beta$-lactam/ $\beta$-lactamase inhibitor \pm erythromycin or other macrolides (1). In our previous retrospective studies $(5,6)$, regarding community-acquired pneumonia in the elderly, S. pneumoniae, methicillin-sensitive Staphylococcus aureus (MSSA), and aerobic gram-negative bacilli were frequently isolated, while Mycoplasma pneumoniae, Chlamydia pneumoniae, Legionella pneumophila, and respiratory viruses were infrequently isolated. Since there have been few reports on the etiology of community-acquired pneumonia in the elderly in Japan, we therefore decided to prospectively study the clinical features, etiology and outcome of pneumonia in elderly patients admitted to our hospital during a two-year period.

\section{Methods}

\section{Patients}

We prospectively evaluated all elderly patients over 65 years old admitted to Kawasaki Medical School Kawasaki Hospital

\footnotetext{
From the Division of Respiratory Diseases, Department of Medicine, Kawasaki Medical School Kawasaki Hospital, Okayama, *the Division of Respiratory Diseases, Department of Medicine, Kawasaki Medical School, Kurashiki and **Kawasaki Medical Welfare University, Matsushima Received for publication July 25, 2000; Accepted for publication February 24, 2001

Reprint requests should be addressed to Dr. Yoshihiro Kobashi, the Division of Respiratory Diseases, Department of Medicine, Kawasaki Medical School Kawasaki Hospital, 2-1-80 Nakasange, Okayama 700-8505
} 
(a 650-bed community general hospital) during the two-year period between April 1998 and March 2000. All patients admitted to this hospital from home had chest radiographs taken. For the purpose of this study, the inclusion criteria for pneumonia were; the presence of new chest radiographic infiltrates on admission and at least two of the following three criteria; history of fever ( $\geqq 37.5^{\circ} \mathrm{C}$ ), leukocytosis ( $\geqq 10,000 / \mu \mathrm{l}$ ), or elevation of C-reactive protein ( $\geqq 1.00 \mathrm{mg} / \mathrm{dl}$ ). Patients with clinical signs of lower respiratory tract infection without chest radiographic confirmation, or patients presenting with opacities (for example; carcinoma) were excluded. Furthermore, patients with severe, chronic illness or disability in whom pneumonia was an expected terminal event were excluded from the study.

Prior to the study, the protocol was approved by the institutional ethics committee and all patients gave their informed consent to participate.

\section{Investigation}

Sputum specimens were mostly obtained before the administration of antibiotics whenever possible. Blood was taken for culture (41 cases, $49 \%$ ) and for a full blood count and differential white cell count; the following analyses were carried out: the erythrocyte sedimentation rate (ESR), C-reactive protein (CRP), serum protein, serum albumin, liver function test, renal function test, immunoglobulin, and purified protein derivative (PPD). Throat swabs were taken for virus and $M$. pneumoniae isolation. Serum was collected both at admission and two or three weeks after admission and tested for complement fixation antibody to influenza virus A and B, respiratory syncytial (RS) virus, adenovirus, parainfluenza 1, 2, 3 viruses, cytomegalovirus, $M$. pneumoniae, $C$. psittaci, and the indirect fluorescent antibody to $L$. pneumophila. Antibodies to $C$. pneumoniae were determined using the enzyme-linked immunosorbent assay (ELISA). Respiratory samples were obtained whenever possible by means of sputum collection (Gram stain and culture) $(n=84)$ or from pleural fluid $(n=5)$. Sputum data were only evaluated when the Gram stain test revealed numerous leukocytes ( $\geqq 25$ in a $100 \times$ microscopic field) and few epithelial cells ( $<10$ in a $100 \times$ microscopic field). The etiology of pneumonia was determined as definite under the following conditions: blood and pleural fluid cultures yielded a pathogen; serological data were considered to indicate recent infection if a four-fold rise in titers was documented between initial and subsequent blood samples or a single titer was elevated to $\geqq \times 256$; valid sputum sample cultures yielded $\geqq$ $10^{7} /$ colony-forming units (CFU); if L. pneumophila. Mycobacterium tuberculosis, C. pneumoniae or M. pneumoniae was isolated from a specimen, the bacterium was considered to be a definitive pathogen even if the culture showed little growth.

\section{Results}

\section{Patient characteristics}

Consecutive patients who appeared to fulfill the criteria for a diagnosis of community-acquired pneumonia were studied.
Fourteen patients were excluded (seven with organizing pneumonia, four with obstructive pneumonia secondary to lung cancer, two with drug-induced pneumonia and one with eosinophilic pneumonia). The remaining 84 patients (50 males and 34 females) who fulfilled the criteria were studied in detail. The mean age $( \pm \mathrm{SD})$ of the patients was $78.0 \pm 7.6$ years (ranging from 65 to 96). All patients came from their own home. Twelve patients had been previous bedridden.

Seventy-six patients (10\%) had at least one underlying disease (Table 1). Thirty-eight patients $(45 \%)$ had a history of respiratory disease, with chronic obstructive pulmonary disease (COPD) being most common (16 cases). Sixty-two patients (74\%) had a history of non-respiratory disease, most commonly cerebrovascular disease (16 patients). Only eight patients had previously been in good health. Twenty-eight patients were current smokers, 22 were ex-smokers, and 15 drank alcohol regularly. Thirty-three patients (39\%) had received prior antibiotics before admission.

Symptoms had been present for 3 to 68 (median: 11) days. Clinical features are recorded in Table 2. Clinical symptoms and physical signs were obtained from 84 patients. The main clinical symptoms were cough in 52 patients, fever in 47 , and sputum in 47 . One-third of the patients had atypical symptoms

Table 1. Clinical Characteristics of 84 Patients with Community-acquired Pneumonia

\begin{tabular}{lc}
\hline Age, years & $78.0 \pm 7.6$ \\
Gender, M/F & $50 / 34$ \\
Residence & \\
Family & $74(88 \%)$ \\
Alone & $10(12 \%)$ \\
Previous physical activity & \\
Bedridden & $12(14 \%)$ \\
Smoking habit & $50(60 \%)$ \\
Current smoker & 28 \\
Ex-smoker & 22 \\
Chronic alcoholism & $15(18 \%)$ \\
Underlying disease & \\
Respiratory disease & $38(45 \%)$ \\
COPD & 16 \\
Old pulmonary tuberculosis & 7 \\
Bronchial asthma & 4 \\
Lung fibrosis & 3 \\
Bronchiectasis & 3 \\
Lung cancer & 2 \\
Other & 3 \\
Non-respiratory disease & $62(74 \%)$ \\
Cerebrovascular disease & 16 \\
Chronic heart disease & 12 \\
Gastrointestinal disease & 12 \\
Chronic liver disease & 5 \\
Psychological disease & 5 \\
Diabetes mellitus & 4 \\
Others & $8(10 \%)$ \\
Previously healthy & \\
\hline
\end{tabular}

COPD: Chronic obstructive pulmonary disease. 
Community-acquired Pneumonia in the Elderly

Table 2. Clinical Symptoms and Physical Signs

\begin{tabular}{llc}
\hline & & $\begin{array}{c}\text { Elderly patients } \\
(84 \text { cases })\end{array}$ \\
\hline Clinical symptoms & Fever $\left(38.0^{\circ} \mathrm{C} \leqq\right)$ & $47(56 \%)$ \\
& Cough & $52(62 \%)$ \\
& Sputum & $47(56 \%)$ \\
& Dyspnea & $16(19 \%)$ \\
& Chest pain & $12(14 \%)$ \\
& Consciousness disturbance & $7(8 \%)$ \\
& Gastrointestinal symptoms & $7(8 \%)$ \\
\hline Physical signs & Temperature $\left(38.0^{\circ} \mathrm{C} \leqq\right)$ & $47(56 \%)$ \\
& Tachypnea $(24 / \mathrm{min} \leqq)$ & $59(70 \%)$ \\
& Tachycardia $(100 / \mathrm{min} \leqq)$ & $58(69 \%)$ \\
& Hypotension $(100 \mathrm{mmHg}>)$ & $15(18 \%)$ \\
& Temperature $\left(35.0^{\circ} \mathrm{C}>\right)$ & $1(1 \%)$ \\
\hline
\end{tabular}

of pneumonia such as dyspnea, consciousness disturbance, and gastrointestinal symptoms. The main physical signs were tachypnea in 59 patients, tachycardia in 58 , high temperature in 47 , hypotension in 15 , and low temperature in 1 .

The main laboratory data at admission were as follows: the white blood cell count was above $10,000 / \mu 1$ in $58 \%$, the ESR was over $20 \mathrm{~mm}$ in one hour in $95 \%$, the CRP concentration was over $1.0 \mathrm{mg} / \mathrm{dl}$ in $100 \%$, serum protein was under $6.5 \mathrm{~g} / \mathrm{dl}$ in $30 \%$, serum albumin was under $3.5 \mathrm{~g} / \mathrm{dl}$ in $35 \%$, cholesterol was under $130 \mathrm{mg} / \mathrm{dl}$ in $27 \%$, cholinesterase was under 240 $\mathrm{IU} / \mathrm{l}$ in $25 \%$, blood urea nitrogen (BUN) was over $20 \mathrm{mg} / \mathrm{dl}$ in $23 \%$, the PPD test was negative in $55 \%$, and serum globulin was under $2.5 \mathrm{~g} / \mathrm{dl}$ in $12 \%$.

Extension of an infiltration shadow due to pneumonia affected one-third of the area of the unilateral lung field in 38\%, the whole unilateral lung field in $52 \%$, and more than the unilateral lung field in $10 \%$.

\section{Etiology of pneumonia}

The causative microorganisms were established in 40 patients (48\%); 16 of these showed evidence of polymicrobial agents; two agents in 10 and three agents in 6 . The noninvasive microbiological tests employed and the results obtained are listed in Tables 3 and 4 . A blood culture was obtained from 41 patients. Three cultures yielded causative microorganisms ( $S$. pneumoniae in $1, K$. pneumoniae in $1, E$. coli in 1$)$. Satisfactory sputum samples were obtained from 64 patients, and 30 samples yielded causative organisms. The cultures of pharyngeal swabs were positive for influenza A virus in one patient.

Serologic tests were performed in 84 patients, with the results meeting the diagnostic criteria in 16 patients. A significant elevation of viral antibody titers in paired sera was observed in 11 patients (influenza A virus 6 , RS virus 3, adenovirus 1 , parainfluenza 1 virus 1 ). However, all these patients had mixed infections (double or triple) with other bacterial infections.

The most common causative microorganisms were $S$.
Table 3. Results of Diagnostic Methods

\begin{tabular}{lcc}
\hline Diagnostic method & $\begin{array}{c}\text { No. of tests } \\
\text { Performed }\end{array}$ & $\begin{array}{c}\text { No. of positive } \\
\text { tests (\%) }\end{array}$ \\
\hline Blood culture & 41 & $3(7 \%)$ \\
Sputum culture & 84 & $30(36 \%)$ \\
Pharyngeal swab & 24 & $1(4 \%)$ \\
Pleural fluid culture & 5 & $1(20 \%)$ \\
Autopsy & 1 & $1(100 \%)$ \\
Serological examination & 84 & $16(19 \%)$ \\
\hline
\end{tabular}

Table 4. Causative Microorganisms

\begin{tabular}{lcc}
\hline Microorganism & No.* & $\%$ \\
\hline Streptococcus pneumoniae & 11 & 13 \\
Respiratory viruses & $11^{\dagger}$ & 13 \\
Haemophilus influenzae & 7 & 8 \\
Mycobacterium tuberculosis & 7 & 8 \\
Klebsiella pneumoniae & 5 & 6 \\
Staphylococcus aureus & 5 & 6 \\
Chlamydia pneumoniae & 3 & 4 \\
Pseudomonas aeruginosa & 2 & 2 \\
Escherichia coli & 1 & 1 \\
Mycoplasma pneumoniae & 1 & 1 \\
Moraxella catarrhalis & 1 & 1 \\
Streptococcus milleri group & 1 & 1 \\
Streptococcus agalactiae & 1 & 1 \\
Anaerobes & 1 & 1 \\
Mycobacterium avium complex & 1 & 1 \\
Unknown & & 52 \\
\hline
\end{tabular}

*Numbers include 11 episodes with polymicrobial agents ( 2 agents: $H$. influenzae + Respiratory virus, $3 ;$ S. pneumoniae + Respiratory virus, 2 ; $S$. aureus + Respiratory virus, $1 ; C$. pneumoniae + Respiratory virus, $1 ; 3$ agents: $P$. aeruginos $a+M$. pneumoniae + Respiratory virus, $1 ; K$. pneumoniae $+S$. aureus + Respiratory virus, $1 ; E$. coli $+P$. aeruginosa+Respiratory virus, $1 ; S$. pneumoniae $+H$. influen$z a e+$ Respiratory virus, 1). Influenza A virus, 6; Respiratory syncytial virus, 3; Adenovirus, 1; Parainfluenza virus 1, 1).

pneumoniae containing penicillin insusceptible $S$. pneumoniae (PISP) found in 11 patients, followed by $H$. influenzae in 7, $M$. tuberculosis in 7, MSSA in 5, C. pneumoniae in 4, and $M$. pneumoniae, M. catarrhalis, Escherichia coli, Streptococcus milleri group, Streptococcus agalactiae, Anaerobes, Mycobacterium avium complex in 1 patient each. However, $L$. pneumophila was not detected (Table 4).

\section{Treatment and prognosis}

The treatment for the elderly patients with community-acquired pneumonia is shown in Table 5. Eleven patients were treated with oral antimicrobial agents, while 73 patients received intravenous antimicrobial agents, either alone for 56 patients or in combination with another antimicrobial agent for 17 patients. Eight patients $(9.5 \%)$ underwent mechanical 
KoBASHI et al

Table 5. Treatment

\begin{tabular}{|c|c|c|c|c|}
\hline \multicolumn{2}{|l|}{ Antimicrobial agents } & \multicolumn{3}{|c|}{$\begin{array}{l}\text { Elderly patients } \\
\qquad(\mathrm{n}=84)\end{array}$} \\
\hline $\begin{array}{l}\text { Oral } 11 \text { cases } \\
\qquad(13 \%)\end{array}$ & & $\begin{array}{l}\text { Cephem } \\
\text { New quinolone } \\
\text { Macrolide } \\
\text { Penicillin }\end{array}$ & $\begin{array}{l}4 \text { cases } \\
3 \text { cases } \\
3 \text { cases } \\
2 \text { cases }\end{array}$ & \\
\hline \multirow{2}{*}{$\begin{array}{c}\text { Intravenous } 73 \text { cases } \\
(87 \%)\end{array}$} & $\begin{array}{c}\text { Alone } 56 \text { cases } \\
(67 \%)\end{array}$ & $\begin{array}{l}\text { Cephem } \\
\text { Carbapenem } \\
\text { Penicillin } \\
\text { Tetracycline }\end{array}$ & $\begin{array}{l}29 \text { cases } \\
21 \text { cases } \\
5 \text { cases } \\
1 \text { case }\end{array}$ & \\
\hline & $\begin{array}{r}\text { Combination } 17 \text { cases } \\
(20 \%)\end{array}$ & \multicolumn{2}{|c|}{$\begin{array}{l}\text { Carbapenem+Macrolide } \\
\text { Lincomycin+Cephem } \\
\text { Carbapenem+Tetracycline } \\
\text { Carbapenem+Aminoglycoside } \\
\text { Cephem+Tetracycline }\end{array}$} & $\begin{array}{l}9 \text { cases } \\
3 \text { cases } \\
3 \text { cases } \\
1 \text { case } \\
1 \text { case }\end{array}$ \\
\hline Mechanical ventilation & & $8 / 84(10 \%)$ & & \\
\hline
\end{tabular}

ventilation. Including six patients with severe pneumonia who received mechanical ventilation, a total of eight patients $(9.5 \%)$ died, with pneumonia being believed to be the major cause of death. Pathogens were detected in six of these patients. Aerobic gram-negative bacilli were cultured in two, S. pneumoniae in one, S. pneumoniae +influenza A virus in one, aerobic gramnegative bacilli+MSSA in one, and $P$. aeruginosa $+M$. pneumoniae+influenza A virus in one.

\section{Discussion}

Through this prospective study we found that the clinical features of community-acquired pneumonia in the elderly were often atypical, with over one-third having prominent non-respiratory symptoms such as dyspnea, consciousness disturbance and gastrointestinal symptoms. However, other features of infection such as fever, tachypnea and tachycardia were present in most patients on admission. In a previous study (7), similar findings were characteristic of community-acquired pneumonia in the elderly. The atypical presentation of pneumonia in the elderly may delay diagnosis, with concomitant delay in the initiation of antibiotic therapy. This delay has been considered to be an important factor in explaining pneumonia's greater mortality rate in the elderly (8).

The proportion of $48 \%$ in whom a pathogen was identified in our series is similar to the proportion in some other series reported in western countries (9-11) with the exception of the study reported by Lieberman et al (12) in which causative pathogens were identified in over $80 \%$ of the patients. No prospective study concerning the pathogen of community-acquired pneumonia restricted to the elderly in Japan has been done, but one prospective study which reported the etiology of community-acquired pneumonia in all hospitalized patients including the elderly was done by Ishida et al (13). They emphasized that the detection rate of causative pathogens was high (61.0\%), and while $S$. pneumoniae was the most common pathogen (23.0\%), followed by $H$. influenzae (7.4\%), M. pneumoniae $(4.9 \%), K$. pneumoniae $(4.3 \%)$, the $S$. milleri group $(3.7 \%)$, Chlamydia pneumoniae (3.4\%) and respiratory viruses $(2.1 \%)$, Legionella pneumophila was only recognized in a few cases $(0.6 \%)$. Regarding the detection rate of causative pathogens, the frequency of the various microorganisms noted in different studies of community-acquired pneumonia has varied with the parameters used for diagnosis or the percentage of prior antibiotics. Although the yield of a noninvasive microbiological test performed in our study, as shown in Table 3, the diagnostic procedure for detecting pneumococcal capsular antigen from various kinds of specimens is popular in hospitals in Western countries (10). Venkatesan et al (10) reported a higher detection rate for the pathogen of $S$. pneumoniae using serum. However, because this technique is not popular in Japan and we could not use this technique nor could Ishida et al (13). They also used other invasive procedures including transthoracic needle aspiration or bronchoscopic examination to increase the detection rate. In the present study, there were several reasons for the lower diagnostic yield. First, prior antibiotic therapy had been given to $39 \%$ of our patients. Second, we could not use an invasive procedure because the patients were elderly and we could not obtain the patient's informed consent. In spite of this, the most common pathogens were S. pneumoniae and respiratory viruses, such as influenza A virus or RS virus, both detected in $13 \%$. Pneumonia with $\mathrm{H}$. influenzae (8\%), M. tuberculosis (8\%), K. pneumoniae (6\%) and MSSA (6\%) were also common. A high percentage of polymicrobial infections was also noted characteristically, occurring in $31 \%$ of elderly patients. There were 11 cases of mixed infection involving both bacterium and virus in our study, as compared with $12 \%$ of elderly patients in another study (9). 
In some studies in Western counties (9-12) undertaken to investigate the causes of community-acquired pneumonia in the elderly, the distribution of causative pathogens appeared to differ from that in younger adults (8), and included $S$. pneumoniae, causing about $50 \%$ of infections, respiratory viruses, $H$. influenzae, $C$. pneumoniae and $L$. pneumophila, However, $M$. pneumoniae was not frequently encountered in elderly patients. Although respiratory viruses such as influenza A virus or RS virus were also emerging pathogens that occupied a higher rank in the etiology of community-acquired pneumonia in the elderly in our study as opposed to the report of Ishida et al (13), the most conspicuous difference between our study and those done overseas was the low frequency of $L$. pneumophila, as was also true in the study of Ishida et al (13). The incidence of L. pneumophila in Japan remains lower than in Western countries despite thorough testing at many institutions in this country (14). The reason L. pneumophila remains rare in Japan is uncertain. Because the detection rate of $L$. pneumophila by only antibody titer has been low, the number of cases detected may increase if a more sensitive urinary antigen test becomes available for screening.

The rate of mortality for pneumonia is higher in the elderly and reaches $20 \%$ in Western countries (15). In the present study, however, the mortality rate was lower than $8 \%$, and there was a relatively good prognosis compared with that in Western countries. The reason for this improvement of the nutritional state by means of intravenous infusion therapy and the performance of early appropriate empiric antibiotic therapy. In the treatment of elderly patients with community-acquired pneumonia, numerous guidelines have been developed by various national bodies with respect to antibiotic therapy, with specific recommendations for community-acquired pneumonia (1-3). Based on the empiric therapy for community-acquired pneumonia in the elderly, $S$. pneumoniae and $H$. influenzae should be covered in patients who have no serious coexisting illness. These can be adequately covered in most situations by antibiotics such as the second-generation cephalosporins and amoxicillinclavulanate. Although in this study the possibility of infection with pathogens such as $C$. pneumoniae and $L$. pneumophila appeared to be lower, erythromycin or new macrolides may be added for patients in whom these organisms are highly suspected. In cases with serious underlying illness, carbapenem or the third-generation cephalosporins may be needed to cover aerobic gram-negative bacilli containing $P$. aeruginosa.

Considering the prevalence of community-acquired pneumonia in relation especially to influenza A virus and S. pneumoniae, an important consideration is the prevention of pneumonia by means such as immunization therapy. In Japan influenza and pneumococcal vaccines have been recently used without provision from the National Health Insurance and have been found to be effective in the elderly $(16,17)$. Because these vaccines are relatively safe (18) and have the potential to prevent serious disease, their routine use in the elderly should be recommended.

In summary, the results of the present study have demon- strated the prevalence of respiratory viruses, such as influenza A virus and RS virus, and the forming of mixed infections with $S$. pneumoniae or $H$. influenzae to be on the increase in community-acquired pneumonia in the elderly. We consider that it might be possible to reduce the high mortality rate of this disease in the elderly by means of routine use of influenza and pneumococcal vaccines in addition to early appropriate antibiotics.

\section{References}

1) Niederman MS, Bass JB Jr, Campbell GD, et al. and American Thoracic Society. Guidelines for the initial management of adults with community-acquired pneumonia: diagnosis, assessment of severity, and the initial antimicrobial therapy. Am Rev Respir Dis 148: 1418-1426, 1993.

2) The British Thoracic Society. Guidelines for the management of community-acquired pneumonia in adults admitted to hospital. Br J Hosp Med 49: 346-350, 1993.

3) Bartlett JG, Breiman RF, Mandell LA, File TM Jr, and The Infectious Diseases Society of America. Community-acquired pneumonia in adults: guidelines for management. Clin Infect Dis 26: 811-838, 1998.

4) Japanese Respiratory Society. Guidelines for the management of community-acquired pneumonia in adults. Nippon Kokyuki Gakkai Zasshi, 2000.

5) Kobashi Y, Fujita K, Karino T, et al. Clinical analysis of pneumonia in the elderly in a community hospital -comparison of community- acquired pneumonia and nosocomial pneumonia. Kansenshogaku Zasshi 73: 884 892, 1999.

6) Kobashi Y, Fujita K, Karino T, et al. Clinical analysis of community-acquired pneumonia requiring hospitalization in a community hospital -comparison of elderly and non-elderly patients. Kansenshogaku Zasshi 74: 43-50, 2000.

7) Feldman C. Pneumonia in the elderly. Clin Chest Med 20: 563-573, 1999.

8) Niederman MS, Fein AM. Community-acquired Pneumonia in the Elderly. Respiratory infections in the elderly. Raven Press, New York, 1991: 44-72.

9) Riquelme R, Torres A, El-Ebiary M, et al. Community-acquired pneumonia in the elderly. A multivariate analysis of risk and prognostic factors. Am J Respir Crit Care Med 154: 1450-1455, 1996.

10) Venkatesan P, Gladman J, Macfarlane JT, et al. A hospital study of community acquired pneumonia in the elderly. Thorax 45: 254-258, 1990.

11) Marrie TJ, Haldane EV, Faulkner RS, Durant H, Kwan C. Communityacquired pneumonia requiring hospitalization. Is it different in the elderly? J Am Geriatr Soc 33: 671-680, 1985.

12) Lieberman D, Schlaeffer F, Porath A. Community-acquired pneumonia in old age: a prospective study of 91 patients admitted from home. Age Ageing 26: 69-75, 1997.

13) Ishida T, Hashimoto T, Arita M, Ito I, Osawa M. Etiology of communityacquired pneumonia in hospitalized patients. a 3-year prospective study in Japan. Chest 114: 1588-1593, 1998.

14) Arakawa M, Inamatsu T, Ezaki $T$, et al. A summary of Legionella pneumonia in Japan from 1979 to 1992. Kankyo Kansen 8: 1-10, 1993.

15) Fine MJ, Smith MA, Carson CA, et al. Prognosis and outcomes of patients with community-acquired pneumonia. A meta-analysis. JAMA 275: 134-141, 1996.

16) Esposito AL. Pneumonia in the Elderly. in: Respiratory Infections. Diagnosis and Management, 3rd ed, Pennington JE, Ed., Raven Press, New York, 1994: 257-274.

17) LaForce FM. Respiratory Infections in the elderly. Prevention of community-acquired and nosocomial pneumonia. in: Respiratory Infections in the Elderly, Niederman MS, Ed., Raven Press, New York, 1991: 349363.

18) Honkanen PO, Keistinen T, Kivela SL. Reactions following administration of influenza vaccine alone or with pneumococcal vaccine to the elderly. Arch Intern Med 156: 205-208, 1996. 